\title{
Predictors of mortality in acute pancreatitis complicated with multidrug-resistant Klebsiella pneumoniae infection
}

Di Wu ${ }^{1 \dagger}$, Junjie Ding ${ }^{1 \dagger}$, Yan Jia ${ }^{1}$, Huanmiao Liu $^{2}$, Jie Xiao ${ }^{2}$ and Jie Peng ${ }^{1 *}$

\begin{abstract}
Background: Multidrug-resistant (MDR) Klebsiella pneumoniae infections, from pancreatic infections to bloodstream infections, influence the mortality of patients with acute pancreatitis (AP) on the condition of limited antibiotic choices. The aim of this study was to investigate the predictor of mortality among AP patients complicated with MDRK. pneumoniae infections.

Methods: Seventy-one AP patients who occurred MDR-K. pneumoniae infections from August 1st, 2016 to August 1st, 2020 were enrolled. MDR-K. pneumoniae was defined as the K. pneumoniae strain non-susceptible to at least one agent in three or more antimicrobial categories. MDR-K. pneumoniae isolates were confirmed by Vitek-2 system. Antibiotic susceptibility test was carried out using a micro broth dilution method. Clinical characteristics and drugresistance rates were retrospectively reviewed, and the predictors of mortality were evaluated by univariate and multivariate analyses.
\end{abstract}

Results: The mortality rate of AP patients complicated with MDR-K. pneumoniae infections reached $46.5 \%$ (33 of 71), and pancreas $(n=53)$ was the most common site of MDR-K pneumoniae strains. The drug resistance rates of MDR-K. pneumoniae were high to 11 of 12 common antibiotics (more than 50.0\%) except of tigecycline (23.9\%). The predictor independently associated with mortality was septic shock (hazard ratio 2.959, 95\% confidence intervals $1.396-6.272$, $P=0.005)$.

Conclusions: More attention should be paid for pancreatic MDR-K. pneumoniae infections among AP patients The predictor for mortality of AP patients complicated with MDR-K. pneumoniae infection is septic shock. Therefore, further clinical investigations should focus on areas against septic shock.

Keywords: Acute pancreatitis, Multidrug-resistant infection, Mortality, Drug-resistance, Predictor

\section{Introduction}

Acute pancreatitis (AP) has been a commonly acute abdominal disease with increasing incidence and mortality rate during the past decade [1]. Infectious complications often develop in the middle-late stages, which are

*Correspondence: pengjie2014@csu.edu.cn

${ }^{\dagger}$ Di Wu and Junjie Ding contributed equally to this work

1 Department of Gastroenterology, Xiangya Hospital, Central South

University, Xiangya Road, Changsha 410008, China

Full list of author information is available at the end of the article the major causes for second death peak of AP. The mortality rate for AP, especially severe AP (SAP) patients, complicated with bacterial infections was up to $70 \%$ [24]. Besides, there were $30 \%$ of AP patients subsequently suffered from (peri)pancreatic or extrapancreatic infections [3].

Due to prolonged hospitalizations and exposure to overused antibiotics, AP patients have become a highrisk population for multidrug-resistant (MDR) bacterial infections [5]. Recently, several studies have showed that 
a rising proportion of AP patients with MDR bacterial infections reached 63\% [5-9]. MDR-K. pneumoniae has a strong drug-resistant ability through several mechanisms including target alteration, drug inactivation, decreased cell permeability, and increased efflux pump activity [1013]. MDR-Klebsiella pneumoniae is found as a dangerous pathogen for hospital-acquired infections because of the limited antibiotic regimens [14-16]. Similarly, some studies have given an account of MDR bacterial infections among AP patients which aimed to differentiate between MDR and non-MDR bacterial infections, but devoted to the aspect of the drug-resistance and predictors for mortality of AP patients complicated with MDR infections $[5,15,17]$. In addition, the availability of regional drugresistance profiles is likely to improve the appropriateness of anti-infective treatments, and the predictors for mortality of AP patients with MDR bacterial infections need to be identified in order to raise the survival rate.

Up to now, there only few case reports have paid attention to the MDR-K. pneumoniae infection in AP, which suggest that the knowledge related to MDR-K. pneumoniae infection among AP patients is still needed to be enhanced [7, 18]. The present bi-centric retrospective cohort study has devoted to investigate the drug-resistance and the predictors of overall mortality among AP patients complicated with MDR-K. pneumoniae infection, thereby making progress in the prognosis in this population.

\section{Methods}

\section{Ethics committee approval}

Due to the property of retrospective cohort study, our study would not interfere with the enrolled patients. Institutional Review Board of Xiangya Hospital (No. 202103047) and the Third Xiangya Hospital (No. 21019) approved the waiver of patient informed consent. This study and all methods have been performed in accordance with the Declaration of Helsinki and obtained ethics approval to collect data from Ethic Committee of the Xiangya Hospital of Central South University (No. 202103047) and IRB of The Third Xiangya Hospital of Central South University (No. 21019). Information was gathered from electrical medical system in an anonymous manner. The authors ensure patient data confidentiality.

\section{Study design and setting}

Seventy-one AP patients who developed MDR-K. pneumoniae infections, in Xiangya Hospital, a 3500-bed tertiary-care teaching hospital, and Third Xiangya Hospital, an 1800-bed tertiary-care teaching hospital, Central South University, Changsha, China, from August 1st, 2016 to August 1st, 2020 were retrospectively enrolled. The follow-up period to observe the clinical outcome lasted for 90 days from the day of onset of MDR-K. pneumoniae infection which was defined as the date of first MDR-K. pneumoniae positive sample collection (not reported time point from microbiology laboratory). Clinical characteristics, from electronical medical system, were recorded within $24 \mathrm{~h}$ after the time of entry into the cohort. Clinical outcomes were divided into mortality and survival to identify the independent predictors.

\section{Patients}

In our cohort, diagnosis and classification of AP were based on the criteria of revised Atlanta classification [19]. Criteria of etiology are listed as follows: (1) biliary: choledocholithiasis or cholelithiasis by enhanced computed tomography; (2) hypertriglyceridemia: triglyceride level $>1000 \mathrm{mg} / \mathrm{dl}$; (3) alcoholic: drinking $>50 \mathrm{~g} / \mathrm{d}$ for at least 1 year. The onset of MDR-K. pneumoniae infection (study entry) indicated to the date of first positive sample collection. In addition, doctors prescribed the empirical antibiotic treatment to 'suspected' infections before getting the further identification and drug-resistance for targeted therapy. The initial diagnoses of 71 patients were moderately SAP and SAP since most mild AP patients were treated in lower-level hospitals. There were 38 patients in Xiangya hospital and 33 patients in Third Xiangya hospital enrolled. We excluded patients $<16$ or $>80$ years old, with a history of chronic pancreatitis or pancreatic surgery, as well as with no positive specimen result.

\section{Treatment protocol of AP}

According to the latest international guidelines Patients were assessed and managed by the multidiscipline team in the early stage of admission, including pancreatic surgeons, radiologists, intensive care unit and gastroenterology physicians. Conservative treatments were taken for (peri)pancreatic necrosis without evidence of secondary infections to intensely observe any changes in the conditions of the patients. Antibiotics (cefoperazone-sulbactam, carbapenems and so on) were previously prescribed when necessary and were adjusted according to the subsequent microbiology profile.

We collected specimens from the 'suspected' infected patients, including (peri)pancreatic necrosis, blood, bronchial alveolar lavage fluid as well as bile, then we immediately transported them to clinical microbiology laboratory for identifications of the causative pathogens. In both 2 tertiary medical centers, MDR-K. pneumoniae pancreatic infection was characterized as the (peri)pancreatic necrosis or fluid obtained with positive specimen from the first therapeutic intervention instead of the fine-needle aspiration. The therapeutic intervention was performed in AP patients, who are complicated 
with clinically confirmed (peri)pancreatic infection after failure of conservative treatment. The step-up approach included percutaneous catheter drainage or endoscopic transluminal drainage and if necessary, subsequent minimal access retroperitoneal necrosectomy, video-assisted retroperitoneal debridement, or endoscopic transluminal necrosectomy and open necrosectomy. In line with the latest international guidelines, treatment for infectious pancreatic necrosis was performed in accordance with patient's condition according to [20].

\section{Definition}

MDR-K. pneumoniae infections were defined with positive specimen results according to the criteria of the Centers for Disease Control [21]. On the basis of the Berlin Definition of acute respiratory distress syndrome, acute respiratory distress syndrome was diagnosed and classified [22]. Diagnostic methods of pancreatic infections included: (1) enhanced computed tomography showing the presence of extraluminal gas, and (2) positive specimen from pancreatic necrosis obtained by first drainage or necrosectomy. Septic shock was diagnosed in the patient who required vasoactive drugs for maintaining mean arterial blood pressure $>65 \mathrm{mmHg}$ with value of serum lactate $>2 \mathrm{mmol} / \mathrm{l}$ after fluid resuscitation with MDR-K. pneumoniae infection [23].

\section{Microbiology}

All specimens were collected by clinicians when patients were suspected for infections or first intervention for infected pancreatic necrosis (pancreatic infections), then we immediately transported specimens to microbiology laboratory in line with standard procedures [24]. Only the data at the first time of positive result was recorded for further statistical analysis when the MDR-K. pneumoniae pathogen occurred repeatedly in the same site of one patient. The specimen was injected into each bottle of a set of aerobic and anaerobic culture bottles and immediately transported to the clinical microbiology laboratory. Identification and antimicrobial susceptibility testing of MDR-K. pneumoniae pathogen were carried out using the Vitek-2 system (bioMe'rieux, Marcy L'etoile, France). Minimum inhibitory concentratio was measured via a micro broth dilution method following the standards of National Committee for Clinical Laboratory. Positive specimen and drug-resistance results were reported at the same time from microbiology laboratory [24]. Breakpoints of minimum inhibitory concentration (susceptible, resistant) for each antibiotics were according to the Clinical Laboratory Standard Institute guidelines: amoxicillin-clavulanate $(\leq 8 / 4, \geq 32 / 16 \mu \mathrm{g} / \mathrm{ml})$, amikacin $(\leq 16, \geq 32 \mu \mathrm{g} / \mathrm{ml})$, aztreonam $(\leq 4, \geq 16 \mu \mathrm{g} / \mathrm{ml})$, ciprofloxacin $(\leq 0.25, \geq 1 \mu \mathrm{g} / \mathrm{ml})$, gentamicin $(\leq 4, \geq 16 \mu \mathrm{g} /$ $\mathrm{ml})$, imipenem $(\leq 2, \geq 8 \mu \mathrm{g} / \mathrm{ml})$, meropenem $(\leq 2, \geq 8 \mu \mathrm{g} /$ $\mathrm{ml})$, levofloxacin $(\leq 0.5, \geq 2 \mu \mathrm{g} / \mathrm{ml})$, sulfamethoxazole $(\leq 2 / 38, \geq 4 / 76 \mu \mathrm{g} / \mathrm{ml})$, piperacillin-tazobactam $(\leq 16 / 4, \geq 128 / 4 \mu \mathrm{g} / \mathrm{ml})$, ceftriaxone $(\leq 8, \geq 64 \mu \mathrm{g} / \mathrm{ml})$ and tigecycline $(\leq 2, \geq 8 \mu \mathrm{g} / \mathrm{ml})$. MDR-K. pneumoniae was defined as the strain non-susceptible to at least one agent in three or more antimicrobial categories [10].

\section{Statistical analysis}

For continuous variables, data were expressed as mean $( \pm \mathrm{SD})$ and compared with Student's t-test or MannWhitney-U test. Categorical variables were compared with the $\chi^{2}$ test or Fisher exact tests. Only the characteristics with value of $P<0.05$ can enter the multivariate analysis. In multivariate analysis, survival time was put into Cox regression analysis to confirm the independent predictors associated with overall mortality. Hazard ratio and $95 \%$ confidence interval were calculated to estimate the degree of proportional hazard. Survival distribution for the independent predictors was described via Kaplan-Meier curve. A value of $P<0.05$ (two-tailed) was defined as statistically significant, and SPSS 24.0 (IBM SPSS Statistics, IBM Corp., Armonk, NY, US) was used for all statistical analysis.

\section{Results}

During the 4-year-study period, 71 AP patients experienced MDR- $K$. pneumoniae infections. The etiology of 71 patients was categorized as biliary $(n=30$, $42.3 \%)$, hyperlipidemic $(n=26,36.6 \%)$, alcoholic $(n=3$, $4.2 \%)$ and other causes $(n=12,16.9 \%)$. The mean age of the patients was 48.8 years. $56(78.9 \%)$ and $15(21.1 \%)$ patients were male and female, respectively. Forty-three patients $(60.6 \%)$ were classified as SAP, and 28 patients (39.4\%) were moderately SAP. Twenty-four (33.8\%) patients met criteria for septic shock, and 27 (38.0\%) patients required invasive mechanical ventilation support. Sixty-three patients $(88.7 \%)$ were referred from other hospitals 2 days after onset of AP with a median referral time of 19.8 days. Fifty-seven patients (80.3\%) occurred multiple organisms or sites infections. Acinetobacter baumannii infections $(\mathrm{n}=33,46.5 \%)$ were most common co-infections, followed by Klebsiella ozaenae $(\mathrm{n}=14,19.7 \%)$, Pseudomonas aeruginosa $(\mathrm{n}=11,15.5 \%)$ and Burkholderia cepacia $(\mathrm{n}=11,15.5 \%)$. The mortality of AP patients complicated with MDR-K. pneumoniae infection was $46.5 \%$ (33 of 71). Percutaneous catheter drainage to minimal access retroperitoneal necrosectomy was most commonly therapeutic intervention $(n=19)$ for pancreatic infections (only 53 patients), follow by step-up to open necrosectomy $(n=10)$, only percutaneous catheter drainage $(n=7)$ or endoscopic transluminal drainage $(n=7)$, open necrosectomy $(n=4)$, percutaneous 
catheter drainage to video-assisted retroperitoneal debridement $(\mathrm{n}=3)$ and endoscopic transluminal drainage to endoscopic transluminal necrosectomy $(n=3)$. The details of clinical characteristics and comparison between alive and death group are presented in Table 1 . In the univariate analysis, multiple infected organisms or sites $(P=0.042)$, SAP $(P=0.003)$, invasive mechanical ventilation $(P<0.001)$, sepsis shock $(P<0.001)$ and hemorrhage $(P<0.001)$ were significantly related to mortality. In the multivariable analysis (Table 2), only septic shock (hazard ratio 2.959, 95\% confidence interval 1.396$6.272, P=0.005)$ was the independent predictor of overall mortality. In Fig. 1, compared with patients without septic shock, the survival rate was significantly lower in the patients suffered from septic shock ( $16.7 \%$ vs. $72.3 \%$, $P<0.001)$.

Drug-resistance rate and distribution of MDR-K pneumoniae strains are shown in Table 3. Pancreas(peri) $(\mathrm{n}=53)$ was the most common site of MDR-K pneumoniae pathogens in AP patients, followed by bloodstream $(n=32)$, lung $(n=27)$ and biliary tract $(n=1)$. Drug-resistance rates of MDR-K pneumoniae to 11 of 12 antibiotics were more than $50 \%$, including amoxicillin-clavulanate, amikacin, aztreonam, ciprofloxacin, gentamicin, imipenem, meropenem, levofloxacin, sulfamethoxazole, piperacillin-tazobactam, ceftriaxone, except tigecycline (23.9\%).

\section{Discussion}

In the past decade, the rapidly emerging MDR pathogens associated infections with limited therapeutic options has become a global threat to medical systems [11, 17]. The crisis of MDR bacterial infection was more frequently occurring in developing countries. K. pneumoniae, as the second most common nosocomial gram-negative bacterium, leads to significantly high all-cause mortality in worldwide [10, 12, 14, 25]. Carbapenem, amikacin and tigecycline used to be effective and latest antibiotics for MDR-K. pneumoniae treatments, but recent researches revealed that drug-resistance rates of these three antibiotics were significantly increasing which may be associated with overuse during treatments $[24,26]$. In this era of MDR bacterial infections, clinician in all departments should pay more attentions on the preventions and treatments of MDR-K. pneumoniae infections [13, 17].

Infection is one of the most common complications in AP patients due to prolonged hospitalization and excessive anti-inflammatory therapy $[1,6,9,27]$. To our knowledge, this is the largest retrospective-cohort-study investigating the predictors of mortality in AP patients complicated with MDR-K. pneumoniae infections. It is consistent with previous studies that biliary etiology was the most common etiology for MDR-K. pneumoniae infections [5, 28]. In line with Ning et al., our study found that there was a higher mortality (46.5\%) of AP patients complicated with MDR-K. pneumoniae infections which confirmed that MDR-K. pneumoniae has played a lethal role in the procedure of AP patients [5, 15].

The main finding of our cohort study is that the independent predictor of mortality among AP patients with MDR-K. pneumoniae infections was septic shock. Immune suppression in early stage of AP may result in excessive systemic inflammatory response syndrome, inducing intestinal barrier dysfunction, thereby increasing intestinal mucosal permeability. Infections of AP patients may be induced by enteric pathogens that translocated into the parenteral system, especially bloodstream, to cause infection due to intestinal barrier disruption [29]. Septic shock is the most severe infectious complication in bloodstream infections. Early recognition and successful management of septic shock remain numerous knowledge gaps and challenges, because SAP patients without infections may mimic septic shock [30]. Patients with septic shock should be timely received antibiotic treatments, because there is a definite guideline to start anti-sepsis shock therapy as early as possible [31]. It is the first report described hazards of septic shock for AP patients complicated MDR-K. pneumoniae infections, which alerts clinicians to prevent barrier disfunction as well as bacterial translocation so as to prompt timely diagnosis and treatment.

Jain et al. revealed that infected pancreatic necrosis infected by MDR bacteria rather than non-MDR bacteria, was an independent predictor of mortality in AP patients, however, it was not significantly related to death in our study [9]. The possible reason might be all our enrolled patients were suffered from MDR bacterial infections with considerable proportion of multiple sites which may reduce the hazard of mono-pancreatic infections in the univariate analysis. The analysis of the intervention for infectious pancreatic necrosis was not the focus of our study which would be done in future.

Consistent with the previous analysis of infections, our microbiologic result demonstrated that bloodstream $(\mathrm{n}=32)$ was the most common extra-pancreatic site of MDR-K. pneumoniae infections which may verify our hypothesis for bacterial translocation to the bloodstream [32]. According to previous studies, drug resistance of MDR-K. pneumoniae pathogens causing AP secondary infections was quite serious $[5,15]$. In our cohort, most strains were carbapenem-resistant $K$. pneumoniae, which may be associated with prophylactic antibiotic treatment and high referral rate [27]. Li et al. reported that carbapenem was considered as the front-line regimen for AP patients with 'suspected' pancreatic infections [17]. By contrast, Tugal et al. oppositely reported that imipenem/ 
Table 1 Clinical characteristics and comparison between survival and mortality of 71 AP patients with MDR-K. pneumoniae infections

\begin{tabular}{|c|c|c|c|c|}
\hline Characteristics & Total & Survival $(n=38)$ & Mortality $(n=33)$ & $P$ \\
\hline Age, years (mean $\pm S D$ ) & $48.8 \pm 12.7$ & $49.5 \pm 11.8$ & $48.1 \pm 13.6$ & 0.632 \\
\hline Sex, n (\%) & & & & 0.571 \\
\hline Male & $56(78.9)$ & $29(76.3)$ & $27(81.8)$ & \\
\hline Female & $15(21.1)$ & $9(23.7)$ & $6(18.2)$ & \\
\hline Etiology, n (\%) & & & & 0.688 \\
\hline Biliary & $30(42.3)$ & $15(39.5)$ & $15(45.5)$ & \\
\hline Hypertriglyceridemia & $26(36.6)$ & $14(36.8)$ & $12(36.4)$ & \\
\hline Alcohol & $3(4.2)$ & $1(2.6)$ & $2(6.1)$ & \\
\hline Others & $12(16.9)$ & $8(21.1)$ & $4(12.1)$ & \\
\hline Classification of AP, n (\%) & & & & $0.003^{*}$ \\
\hline Moderately severe AP & $28(39.4)$ & $21(55.3)$ & $7(21.2)$ & \\
\hline Severe AP & $43(60.6)$ & $17(44.7)$ & $26(78.8)$ & \\
\hline Primary site of infections, n (\%) & & & & 0.744 \\
\hline Pancreas (peri) & $53(74.6)$ & $29(76.3)$ & $24(72.7)$ & \\
\hline Bloodstream & $9(12.7)$ & $4(10.5)$ & $5(15.2)$ & \\
\hline Lung & $8(11.3)$ & $4(10.5)$ & $4(12.1)$ & \\
\hline Biliary tract & $1(1.4)$ & $1(2.6)$ & 0 & \\
\hline Multiple infected organisms or sites, $\mathrm{n}(\%)$ & $57(80.3)$ & $27(71.1)$ & $30(90.9)$ & $0.042^{*}$ \\
\hline \multicolumn{5}{|l|}{ Concomitant infected bacteria } \\
\hline Acinetobacter baumannii & $33(46.5)$ & $16(42.1)$ & $17(51.5)$ & 0.428 \\
\hline Klebsiella ozaenae & $14(19.7)$ & $5(13.2)$ & $9(27.3)$ & 0.136 \\
\hline Pseudomonas aeruginosa & $11(15.5)$ & $7(18.4)$ & $4(12.1)$ & 0.464 \\
\hline Burkholderia cepacia & $11(15.5)$ & $4(10.5)$ & $7(21.2)$ & 0.215 \\
\hline Escherichia coli & $8(11.3)$ & $6(15.8)$ & $2(6.1)$ & 0.359 \\
\hline Proteus mirabilis & $5(7.0)$ & $4(10.5)$ & $1(3.0)$ & 0.363 \\
\hline Enterobacter cloacae & $5(7.0)$ & $1(2.6)$ & $4(12.1)$ & 0.176 \\
\hline Referred patient, $n(\%)$ & $63(88.7)$ & $34(89.5)$ & $29(87.9)$ & 1.000 \\
\hline Time of infection from onset of AP, $n(\%)$ & & & & 0.290 \\
\hline$<14$ days & $6(8.5)$ & $5(13.2)$ & $1(3.0)$ & \\
\hline $15-30$ days & $29(40.8)$ & $14(36.8)$ & $15(45.5)$ & \\
\hline$>30$ days & $36(50.7)$ & $19(50.0)$ & $17(51.5)$ & \\
\hline Respiratory function, $\mathrm{n}(\%)$ & & & & 0.811 \\
\hline Mild ARDS & $61(85.9)$ & $33(86.8)$ & $28(84.8)$ & \\
\hline Severe and moderate ARDS & $10(14.1)$ & $5(13.2)$ & $5(15.2)$ & \\
\hline Sepsis shock, n (\%) & $24(33.8)$ & $4(10.5)$ & $20(60.6)$ & $<0.001^{*}$ \\
\hline Invasive mechanical ventilation, $\mathrm{n}(\%)$ & $27(38.0)$ & $7(18.4)$ & $20(60.6)$ & $<0.001^{*}$ \\
\hline Antibiotic therapy, n (\%) & & & & 0.386 \\
\hline Carbapenem (high dose, extended infusion) & $25(35.2)$ & $14(36.8)$ & $11(33.3)$ & \\
\hline Tigecycline & $6(8.5)$ & $3(7.9)$ & $3(9.1)$ & \\
\hline Penicillins/ $\beta$-lactamase inhibitors & $6(8.5)$ & $6(15.8)$ & 0 & \\
\hline Quinolone & $4(5.6)$ & $3(7.9)$ & $1(3.0)$ & \\
\hline Carbapenem and tigecycline & $21(29.6)$ & $6(15.8)$ & $15(45.5)$ & \\
\hline Carbapenem and penicillins/ $\beta$-lactamase inhibitors & $4(5.6)$ & $4(10.5)$ & 0 & \\
\hline Carbapenem and sulphonamides & $3(4.2)$ & $1(2.6)$ & $2(6.1)$ & \\
\hline Polymyxins and fosfomycin & $2(2.8)$ & $1(2.6)$ & $1(3.0)$ & \\
\hline Hospitalization, days (mean \pm SD) & $45.8 \pm 28.8$ & $41.2 \pm 33.3$ & $49.9 \pm 24.1$ & 0.214 \\
\hline \multicolumn{5}{|l|}{ Major complications, n (\%) } \\
\hline Hemorrhage & $23(32.4)$ & $4(10.5)$ & $19(57.6)$ & $<0.001^{*}$ \\
\hline I ntestinal leakage & $15(21.1)$ & $5(13.2)$ & $10(30.3)$ & 0.078 \\
\hline Pancreatic fistula & $8(11.3)$ & $5(13.2)$ & $3(9.1)$ & 0.716 \\
\hline
\end{tabular}


Table 1 (continued)

SD standard deviation, $A P$ acute pancreatitis, $A R D S$ acute respiratory distress syndrome

Table 2 Multivariate analysis of predictors for mortality in AP patients complicated with MDR-K. pneumoniae infections

\begin{tabular}{lll}
\hline Variable & HR $(\mathbf{9 5} \%$ Cls $)$ & $P$ \\
\hline SAP & $2.182(0.866-5.502)$ & 0.098 \\
Male & $0.677(0.257-1.786)$ & 0.431 \\
Age > 50 years & $1.335(0.609-2.926)$ & 0.471 \\
Multiple infected organisms or sites & $1.366(0.394-4.742)$ & 0.623 \\
Hemorrhage & $1.315(0.607-2.847)$ & 0.487 \\
Invasive mechanical ventilation & $1.961(0.896-4.295)$ & 0.092 \\
Septic shock & $2.959(1.396-6.272)$ & 0.005 \\
\hline
\end{tabular}

$\mathrm{HR}$ hazard ratio, $\mathrm{Cl}$ confidence interval

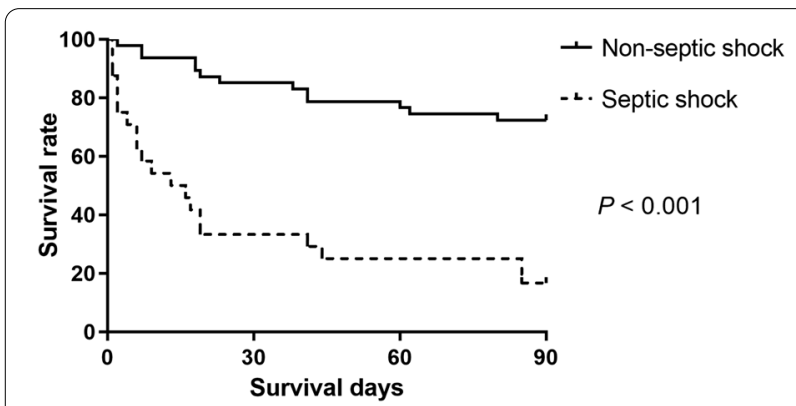

Fig. 1 Kaplan-Meier curve estimated survival rates between septic shock and non-septic shock group (16.7\% vs. $72.3 \%, P<0.001)$ cilastatin was failed to treat a carbapenem-resistant $K$. pneumoniae infected necrotizing pancreatitis[7]. Concerning this conflicting issue, our results suggested that carbapenems (regular dose) may not be effective antibiotics against MDR-K. pneumoniae due to the high drugresistance rate and augmented renal clearance [33, 34]. Prophylactic use of antibiotics, especially carbapenems, may not be recommended in AP for the prevention of infectious complication [20]. MDR-K. pneumoniae has become a growing threat, and it is necessary for clinicians to enhance the administration of antibiotics. However, the phenomenon of overuse and misuse of antibiotics for AP, perhaps resulting in drug-resistant bacteria, has soared worldwide, especially in China [35].

Similar as Moka et al. reported, our cohort showed that tigecycline is recommended as one of last-line antibiotics, whereas carbapenem may be substituted by tigecycline for MDR-K. pneumoniae infection treatment [15]. However, it was reported that tigecycline was overuse with risk of increasing drug-resistance rate in China [24]. Though polymyxin played an essential role in the antibiotic treatment for MDR or extensively drug-resistant bacterial infections, polymyxin resistance assays were lacked for most of patients in our study [36]. Ceftazidime avibactam, reported with better effects than tigecycline, has been approved, by Chinese Food and Drug

Table 3 Resistance rates of 113 MDR-K.pneumoniae strains to 12 antibiotics according to the different sites of infections

\begin{tabular}{|c|c|c|c|c|c|}
\hline Antimicrobial & $\begin{array}{l}\text { Pancreas (peri) } \\
(n=53)\end{array}$ & Bloodstream $(n=32)$ & Lung $(n=27)$ & Biliary tract $(n=1)$ & $\begin{array}{l}\text { Total } \\
\text { strains } \\
(n=113)\end{array}$ \\
\hline Amoxicillin-clavulanate & $53(100)$ & $32(100)$ & $26(96.3)$ & $1(100)$ & $112(99)$ \\
\hline Amikacin & $28(52.8)$ & $18(56.2)$ & $17(63)$ & $0(0)$ & $63(55.8)$ \\
\hline Aztreonam & $45(84.9)$ & $29(90.6)$ & $26(96.3)$ & $1(100)$ & $101(89.4)$ \\
\hline Ciprofloxacin & $44(83)$ & 29 (90.6) & $24(88.9)$ & $1(100)$ & $98(86.7)$ \\
\hline Gentamicin & $39(73.6)$ & $26(81.3)$ & 25 (92.6) & $0(0)$ & $90(79.6)$ \\
\hline Imipenem & $38(71.7)$ & $26(81.3)$ & $24(88.9)$ & $1(100)$ & $89(78.8)$ \\
\hline Meropenem & $38(71.7)$ & $26(81.3)$ & $23(85.2)$ & $1(100)$ & $88(77.9)$ \\
\hline Levofloxacin & $42(79.2)$ & 29 (90.6) & 25 (92.6) & $1(100)$ & $97(85.8)$ \\
\hline Sulfamethoxazole & $20(37.7)$ & $14(43.8)$ & $11(40.7)$ & $1(100)$ & $67(59.3)$ \\
\hline Piperacillin-tazobactam & $41(77.4)$ & 29 (90.6) & $24(88.9)$ & $1(100)$ & $95(84.1)$ \\
\hline Ceftriaxone & $46(86.8)$ & $30(93.8)$ & $27(100)$ & $1(100)$ & $104(92.0)$ \\
\hline Tigecycline & $15(28.3)$ & $7(21.9)$ & $5(18.5)$ & $0(0)$ & 27 (23.9) \\
\hline
\end{tabular}

Values are no. (\%) of resistant strains, except as indicated 
Administration, to treat with MDR-K. pneumoniae infections during recent years [37]. Prescribing combined or novel antibiotic therapy to all the MDR-K. pneumoniae infections, including extensively-drug-resistant or pan-drug-resistant $K$. pneumoniae, is not a reasonable therapeutic option due to the risk of increasing resistance. In this era of MDR, further steps are advised to facilitate molecular or pharmacologic research to potentially accelerate the process of finding novel antimicrobial breakpoints and clearly reveal the mechanism of drug resistance. Furthermore, management of antibiotics would affect drug-resistances of pathogens. However, it may be too difficult to analyze the data on the antibiotic therapy into several subgroups because of limited number of patients which need to be revealed with standardized regimens in the prospective studies.

This double-center retrospective cohort study has several limitations. Firstly, due to its retrospective nature, our study was limited by nonincluded factors, insufficient information as well as selection biases. As the patients in two large tertiary hospital in Central China, most of patients were referred from other hospitals without precise details of antibiotic exposures before referral which may have impact on the outcomes and need to be verified in prospective multi-center studies. Secondly, in the era of highly advanced medical technologies, the novel molecular technology such as third generation sequencing might be used to promote rapid diagnoses and reveal the mechanism of MDR-K. pneumoniae infections. Thirdly, it's noted that the comparison of intervention for infected pancreatic necrosis was lacked, because some patients were only diagnosed with proven extrapancreatic infections. What's more, our analysis only investigated the predictors of overall mortality instead of infection-related mortality as a result of limited number with no ability for excluding the death of polymicrobial infections or major complications which were included as potential predictors. Finally, our findings should be carefully interpreted in the lowly drug-resistant region. Notably, the 4-year-period in two large hospitals in China is the huge advantage of our cohort.

\section{Conclusions}

MDR-K. pneumoniae infection is recognized as a serious complication with high mortality among AP patients. MDR-K. pneumoniae pathogens, most frequently found in (peri)pancreas, are highly resistant to common antibiotics except for tigecycline. We firstly reveal that the independent predictor for the mortality of AP patients complicated with is septic shock. Further clinical investigations should focus on areas against septic shock.
Abbreviations

AP: Acute pancreatitis; SAP: Severe acute pancreatitis; MDR:

Multidrug-resistant.

\section{Acknowledgements}

We thank Prof. Junxia Yan for her help in verifying the statistical analysis in this manuscript.

\section{Authors' contributions}

JP, DW, JJD wrote the main manuscript text and YJ, HML and JX prepared Tables 1, 2, 3 and Fig. 1. All authors reviewed the manuscript. All authors read and approved the final manuscript.

\section{Funding}

This work was supported by Fundamental Research Funds for the Central Universities of Central South University (2021zzts0347), Hunan Provincial Innovation Foundation For Postgraduate, National Natural Science Foundation of China (Grant No. 81670589) and National Natural Science Foundation of China (Grant No. 82170661).

\section{Availability of data and materials}

The datasets used and/or analyzed during the current study are available from the corresponding author on reasonable request.

\section{Declarations}

Ethics approval and consent to participate

Due to nature of retrospective cohort study, our study would not interfere with the enrolled patients. Institutional Review Board of Xiangya Hospital (No. 202103047) and the Third Xiangya Hospital (No. 21019) approved the waiver of patient informed consent. This study and all methods have been performed in accordance with the Declaration of Helsinki and obtained ethics approval to collect data from Ethic Committee of the Xiangya Hospital of Central South University (No. 202103047) and IRB of The Third Xiangya Hospital of Central South University (No. 21019). Information was anonymously analyzed and obtained from electrical medical system. The authors guarantee patient data confidentiality.

\section{Consent for publication}

Not applicable.

Competing interests

The authors declare no competing interests.

\section{Author details}

'Department of Gastroenterology, Xiangya Hospital, Central South University, Xiangya Road, Changsha 410008, China. ${ }^{2}$ Emergency Department, Third

Xiangya Hospital, Central South University, Changsha, China.

Received: 22 May 2021 Accepted: 15 September 2021

Published online: 20 September 2021

References

1. Forsmark C, Vege SS, Wilcox CM. Acute pancreatitis. New Engl J Med. 2017;376(6):596-9.

2. Werge M, Novovic S, Schmidt PN, Gluud LL. Infection increases mortality in necrotizing pancreatitis: a systematic review and meta-analysis. Pancreatology. 2016;16(5):698-707.

3. Brown LA, Hore TA, Phillips ARJ, Windsor JA, Petrov MS. A systematic review of the extra-pancreatic infectious complications in acute pancreatitis. Pancreatology. 2014;14(6):436-43.

4. Pando E, Alberti P, Hidalgo J, Vidal L, Dopazo C, Caralt M, Blanco L, Gómez-Gavara C, Bilbao I, Balsells J, et al. The role of extra-pancreatic infections in the prediction of severity and local complications in acute pancreatitis. Pancreatology. 2018;18(5):486-93. 
5. Ning C, Huang G, Shen D, Bonsu AAFK, Ji L, Lin C, Cao X, Li J. Adverse clinical outcomes associated with multidrug-resistant organisms in patients with infected pancreatic necrosis. Pancreatology. 2019;19(7):935-40.

6. Lee H, Lee SK, Park DH, Lee SS, Seo D, Kim M, Chong YP. Emergence of multidrug resistant infection in patients with severe acute pancreatitis. Pancreatology. 2014;14(6):450-3.

7. Tugal D, Lynch M, Hujer AM, Rudin S, Perez F, Bonomo RA. Multi-drugresistant Klebsiella pneumoniae pancreatitis: a new challenge in a serious surgical infection. Surg Infect. 2015;16(2):188-93.

8. Párniczky A, Kui B, Szentesi A, Balázs A, Szúcs Á, Mosztbacher D, Czimmer J, Sarlós P, Bajor J, Gódi S, et al. Prospective, multicentre, nationwide clinical data from 600 cases of acute pancreatitis. PLoS ONE. 2016;11(10):e165309.

9. Jain S, Mahapatra SJ, Gupta S, et al. Infected pancreatic necrosis due to multidrug-resistant organisms and persistent organ failure predict mortality in acute pancreatitis. Clin Transl Gastroen. 2018;9(10):190-8.

10. Kareem SM, Al-kadmy IM, Kazaal SS, Mohammed Ali AN, Aziz SN, Makharita RR, Algammal AM, Al-Rejaie S, Behl T, Batiha GE, et al. Detection of gyrA and parC mutations and prevalence of plasmid-mediated quinolone resistance genes in Klebsiella pneumoniae. Infect Drug Resist. 2021;14:555-63.

11. Qureshi S, Maria N, Zeeshan M, Irfan S, Qamar FN. Prevalence and risk factors associated with multi-drug resistant organisms (MDRO) carriage among pediatric patients at the time of admission in a tertiary care hospital of a developing country. A cross-sectional study. BMC Infect Dis. 2021;21(1):547.

12. Massinga AJ, Garrine M, Messa A, Nobela NA, Boisen N, Massora S, Cossa A, Varo R, Sitoe A, Hurtado JC, et al. Klebsiella spp. cause severe and fatal disease in Mozambican children: antimicrobial resistance profile and molecular characterization. BMC Infect Dis. 2021;21(1):526.

13. Galani I, Karaiskos I, Giamarellou H. Multidrug-resistant Klebsiella pneumoniae: mechanisms of resistance including updated data for novel $\beta$-lactam- $\beta$-lactamase inhibitor combinations. Expert Rev Anti-Infe. 2021. https://doi.org/10.1080/14787210.2021.1924674.

14. Wu D, Huang X, Jia C, Liu J, Wan Q. Clinical Manifestation, Distribution, and Drug Resistance of Pathogens Among Abdominal Solid Organ Transplant Recipients With Klebsiella pneumoniae Infections. Transpl Proc. 2020;52(1):289-94

15. Moka P, Goswami P, Kapil A, Xess I, Sreenivas V, Saraya A. Impact of antibiotic-resistant bacterial and fungal infections in outcome of acute pancreatitis. Pancreas. 2018;47(4):489-94.

16. Amanati A, Sajedianfard S, Khajeh S, Ghasempour S, Mehrangiz S, Nematolahi S, Shahhosein Z. Bloodstream infections in adult patients with malignancy, epidemiology, microbiology, and risk factors associated with mortality and multi-drug resistance. BMC Infect Dis. 2021;21 (1):636.

17. Li X, Li L, Liu L, Hu Y, Zhao S, Sun J, Wang G, Hai X. Risk factors of multidrug resistant pathogens induced infection in severe acute pancreatitis. Shock. 2020;53(3):293-8

18. Li C, Ma G, Yang T, Wen X, Qin C, Yue L, Jia X, Shen Y, Lu D, Wang L, et al. A rare carbapenem-resistant hypervirulent $\mathrm{K} 1 / \mathrm{ST} 1265$ Klebsiella pneumoniae with an untypeable blaKPC-harboured conjugative plasmid. J Glob Antimicrob Re. 2020;22:426-33.

19. Banks PA, Bollen TL, Dervenis C, Gooszen HG, Johnson CD, Sarr MG, Tsiotos GG, Vege SS. Classification of acute pancreatitis-2012: revision of the Atlanta classification and definitions by international consensus. Gut. 2012;62(1):102-11.

20. IAP/APA evidence-based guidelines for the management of acute pancreatitis. Pancreatology 2013, 13(4):e1-e15.

21. Horan TC, Andrus M, Dudeck MA. CDC/NHSN surveillance definition of health care-associated infection and criteria for specific types of infections in the acute care setting. Am J Infect Control. 2008;36(5):309-32.
22. Ranieri VM, Rubenfeld GD, Thompson BT, Ferguson ND, Caldwell E, Fan E, Camporota L, Slutsky AS. Acute respiratory distress syndrome. JAMA. 2012;307(23):2526-33.

23. Shankar-Hari M, Phillips GS, Levy ML, Seymour CW, Liu VX, Deutschman CS, Angus DC, Rubenfeld GD, Singer M. Developing a new definition and assessing new clinical criteria for septic shock. JAMA. 2016;315(8):775.

24. Wu D, Chen C, Liu T, Jia Y, Wan Q, Peng J. Epidemiology, susceptibility, and risk factors associated with mortality in carbapenem-resistant gram-negative bacterial infections among abdominal solid organ transplant recipients: a retrospective cohort study. Infect Dis Ther . 2021;10(1):559-73.

25. Wu D, Chen C, Liu T, Wan Q. Risk factors for acquisition of carbapenemresistant Klebsiella pneumoniae and mortality among abdominal solid organ transplant recipients with K. pneumoniae infections. Med Sci Monitor. 2020;26:e922996.

26. Wu D, Xiao J, Ding J, Jia Y, Guo Z, Liu H, Peng J. Predictors of mortality and drug resistance among carbapenem-resistant enterobacteriaceaeinfected pancreatic necrosis patients. Infect Dis Ther. 2021;10(3):1665-76.

27. Lu J, Cao F, Ding Y, Wu Y, Guo Y, Li F. Timing, distribution, and microbiology of infectious complications after necrotizing pancreatitis. World J Gastroentero. 2019;25(34):5162-73.

28. Zhu Y, Pan X, Zeng H, He W, Xia L, Liu P, Zhu Y, Chen Y, Lv N. A study on the etiology, severity, and mortality of 3260 patients with acute pancreatitis according to the revised Atlanta classification in Jiangxi, China over an 8-year period. Pancreas. 2017;46(4):504-9.

29. Fishman JE, Levy G, Alli V, Zheng X, Mole DJ, Deitch EA. The intestinal mucus layer is a critical component of the gut barrier that is damaged during acute pancreatitis. Shock. 2014;42(3):264-70.

30. Melo J, Peters JI. Low systemic vascular resistance: differential diagnosis and outcome. CRIT CARE. 1999;3(3):71.

31. Rhodes A, Evans LE, Alhazzani W, Levy MM, Antonelli M, Ferrer R, Kumar A, Sevransky JE, Sprung CL, Nunnally ME, et al. Surviving sepsis campaign: international guidelines for management of sepsis and septic shock: 2016. Intens Care Med. 2017;43(3):304-77.

32. Garret C, Canet E, Corvec S, Boutoille D, Péron M, Archambeaud I, Le Thuaut $\mathrm{A}$, Lascarrou J, Douane F, Lerhun M, et al. Impact of prior antibiotics on infected pancreatic necrosis microbiology in ICU patients: a retrospective cohort study. Ann Intensive Care. 2020;10(1):82.

33. Tian $H$, Chen L, Wu X, Li F, Ma Y, Cai Y, Song S. Infectious complications in severe acute pancreatitis: pathogens, drug resistance, and status of nosocomial infection in a University-Affiliated Teaching Hospital. Digest Dis Sci. 2020;65(7):2079-88.

34. Schwender BJ, Gordon SR, Gardner TB. Risk factors for the development of intra-abdominal fungal infections in acute pancreatitis. Pancreas. 2015:44(5):805-7.

35. Párniczky A, Lantos T, Tóth EM, Szakács Z, Gódi S, Hágendorn R, Illés D, Koncz B, Márta K, Mikó A, et al. Antibiotic therapy in acute pancreatitis: from global overuse to evidence based recommendations. Pancreatology. 2019;19(4):488-99.

36. Mazuski JE, Tessier JM, May AK, Sawyer RG, Nadler EP, Rosengart MR, Chang PK, O'Neill PJ, Mollen KP, Huston JM, et al. The surgical infection society revised guidelines on the management of intra-abdominal infection. Surg Infect. 2017;18(1):1-76.

37. van Duin D, Lok JJ, Earley M, Cober E, Richter SS, Perez F, Salata RA, Kalayjian RC, Watkins RR, Doi Y, et al. Colistin versus ceftazidimeavibactam in the treatment of infections due to carbapenem-resistant enterobacteriaceae. Clin Infect Dis. 2018;66(2):163-71.

\section{Publisher's Note}

Springer Nature remains neutral with regard to jurisdictional claims in published maps and institutional affiliations. 